https://helda.helsinki.fi

\title{
Abstraction in ecology : reductionism and holism as complementary heuristics
}

\section{Raerinne, Jani}

2018-10

Raerinne , J 2018 , ' Abstraction in ecology : reductionism and holism as complementary

heuristics ' , European Journal for Philosophy of Science , vol. 8 , no. 3 , pp. 395-416 . https://doi.org/10.1007/s1319

http://hdl.handle.net/10138/309082

https://doi.org/10.1007/s13194-017-0191-3

unspecified

acceptedVersion

Downloaded from Helda, University of Helsinki institutional repository.

This is an electronic reprint of the original article.

This reprint may differ from the original in pagination and typographic detail.

Please cite the original version. 


\title{
Abstraction in Ecology: Reductionism and Holism as Complementary Heuristics
}

\author{
European Journal for Philosophy of Science (forthcoming) \\ Jani Raerinne \\ Department of Political and Economic Studies, University of Helsinki, Finland \\ E-mail: jani.raerinne@helsinki.fi
}

\begin{abstract}
In addition to their core explanatory and predictive assumptions, scientific models include simplifying assumptions, which function as idealizations, approximations, and abstractions. There are methods to investigate whether simplifying assumptions bias the results of models, such as robustness analyses. However, the equally important issue - the focus of this paper - has received less attention, namely, what are the methodological and epistemic strengths and limitations associated with different simplifying assumptions. I concentrate on one type of simplifying assumption, the use of mega parameters as abstractions in ecological models. First, I argue that there are two kinds of mega parameters qua abstractions, sufficient parameters and aggregative parameters, which have gone unnoticed in the literature. The two are associated with different heuristics, holism and reductionism, which many view as incompatible. Second, I will provide a different analysis of abstractions and the associated heuristics than previous authors. Reductionism and holism and the accompanying abstractions have different methodological and epistemic functions, strengths, and limitations, and the heuristics should be viewed as providing complementary research perspectives of cognitively limited beings. This is then, third, used as a premise to argue for epistemic and methodological pluralism in theoretical ecology. Finally, the presented taxonomy of abstractions is used to comment on the current debate whether mechanistic accounts of explanation are compatible with the use of abstractions. This debate has suffered from an abstract discussion of abstractions. With a better taxonomy of abstractions the debate can be resolved.
\end{abstract}

Key words: abstraction, aggregation, holism, mechanism, pluralism, reductionism, robustness, sufficient parameters

\section{Introduction}

In addition to their core explanatory or predictive assumptions, models make use of simplifying assumptions, which function as idealizations, approximations, and abstractions. Simplifying assumptions make false, unrealistic, or inaccurate presumptions of target systems and/or core assumptions. ${ }^{1}$

The function of simplifying assumptions is to facilitate the modeling and understanding of phenomena. However, the risk is that simplifying assumptions bias the results of models. Robustness analyses provide a method to investigate whether these "dubious details" affect the results of models (Levins 1966; Weisberg and Reisman 2008; Kuorikoski et al. 2010; Raerinne 2013b).

Despite the fact that there are methods to investigate whether simplifying assumptions have effects on the results of models and despite extensive discussion on these methods, an equally important issue - the focus of this paper - has

\footnotetext{
${ }^{1}$ Dividing modeling assumptions into core and simplifying assumptions is simplistic, but accurate enough for the purposes of this paper: I shall be concerned with one type of simplifying assumption only, the use of abstractions in models. Moreover, and due to the reason just mentioned, I shall not discuss how abstractions differ from other simplifying assumptions. This topic is beyond the scope of this paper. For more fine-grained taxonomies of modeling assumptions, see Musgrave (1981), Mäki (2000), Weisberg and Reisman (2008), and Kuorikoski et al. (2010).
} 
received less attention, namely, what are the methodological and epistemic strengths and limitations associated with different simplifying assumptions? Thus, rather than investigating whether simplifying assumptions have effects on the results of models simpliciter, as in robustness analyses, I investigate how and under what conditions the simplifying assumptions are - or are not - useful.

I focus on one variety of simplifying assumption, the use of parameters as abstractions in models. The parameters I discuss are called by different terms in the literature, such as macro, mega, gross-level, or summary parameters or variables. To avoid a plurality of terms, I will employ the term mega parameter. ${ }^{2}$ I argue that there are two kinds of mega parameters qua abstractions that are covered by the term: sufficient parameters and aggregative parameters. The two are associated with different heuristics, holism and reductionism, which many view as incompatible, such as being explanatory rivals, contrary, or even contradictory (see Levins 1998, 2006 and Winther 2006 below).

Aggregation is accompanied by reductionistic research strategies (Wimsatt 1986), whereas sufficient parameters are associated with holistic research strategies (Lane et al. 1976). The two have different functions as abstractions and different sources of errors as well.

In the case of a sufficient parameter, one is investigating whether different lower-level or finer-scale causes have similar or robust effects. If they have similar effects, then a modeler forms a sufficient parameter, which is used in models instead of different specific causes. Sufficient parameters function as abstract, general, or higher-level causal or explanatory surrogates for different lower-level or finer-scale causes or mechanisms that have robust or similar effects in models of a common phenomenon. If a result is robust, the job of identifying the actual cause or mechanism can be irrelevant for some modeling purposes. The examples of sufficient parameters discussed in this paper include resources and environmental heterogeneity (see section 2 ).

In aggregation, one is integrating different lower-level or finer-scale causal components with different or similar effects to extrapolate what their combined effect would be. A modeler forms a parameter, which combines the effects of different causal components into a single monadic coarse scale component, the aggregative parameter, which involves abstracting away the details of the organization of the system. The aggregative parameter is then used in a model as a causal or explanatory surrogate for the different causal components and their organization abstracted away in the parameter. The example of an aggregative parameter discussed in this paper is the total predation response (see section 3 ).

Levins $(1998,2006)$ and Winther (2006) view different research heuristics as contradictory and their synthesis as the true or correct perspective. I will provide a different analysis of abstractions and the associated heuristics. My argument for the complementarity of different abstractions and heuristics is epistemic and methodological. Another difference between my paper and previous authors is that the authors have failed to distinguish between sufficient and aggregate parameters, which are different as abstractions.

\footnotetext{
${ }^{2}$ Many examples of mega parameters are variables (e.g. the total predation response in section 3 ). It is the difference between different simplifying assumptions qua abstractions, rather than the difference between parameters and variables, that is important in this context. However, the discussion of abstractions is historically connected to parameters by Levins and later authors, so I use this term even in the case of variables to avoid unnecessary terminological complications.
} 
I use reductionism to refer to the call for mechanistic models and explanations for ecological phenomena rather than the call for Nagelian theory reduction of ecology to more basic sciences. This is how ecologists understand the difference between holism and reductionism as modeling heuristics. ${ }^{3}$ Note that the two have different goals. Nagelian theory reduction amounts to explaining the higher-level laws and phenomena in terms of lower-level laws, but not to epistemic or methodological elimination of higher-level laws, theories, or phenomena. However, mechanistic reductionism in its extreme form (e.g. "ecological systems are nothing but mere aggregates") would amount to epistemic and methodological elimination in and of ecology. That is, it would amount to the replacement, not reduction, of ecological theories. I am not aware of an author who has explicitly defended an extreme version of mechanistic reductionism in ecology. Schoener (1986) comes close to it, even though he erroneously thinks of himself as defending Nagelian theory reduction in ecology (see section 3 ).

I shall be concerned with models that are used to generate explanations and predictions (see Odenbaugh 2005 for other functions of ecological models). There is a current debate whether - and/or to what extent - mechanistic accounts of explanation are compatible with the use of abstraction, that is, whether mechanistic accounts are committed to the thesis that de-abstracting or adding more details to an explanation always or typically makes the explanation better (see Machamer et al. 2000, 15-18; Kaplan 2011, 347-348; Levy and Bechtel 2013; Boone and Piccini 2016, 1517-1519; and Miłkowski 2016). This debate has suffered from an abstract discussion of abstractions. As I will show, there are different kinds of abstractions, which are utilized by both holists and reductionists. With a functional taxonomy of abstractions, the debate can thus be resolved. Both adding certain kinds of details (i.e. de-abstracting) and omitting certain other kinds of details (i.e. abstracting) can improve mechanistic explanations.

In sections 2 and 3, I discuss methodological functions, strengths, and limitations associated with sufficient and aggregative parameters. In section 4, I argue for epistemic and methodological pluralism in theoretical ecology. Mechanistic and holistic models can both be true of a common phenomenon and be complementary, but have different epistemic strengths and limitations. Moreover, even if they applied to different phenomena, reductionism and holism can nevertheless be viewed as complementary. The two have different methodological strengths and limitations; and we often need another modeling heuristic to see the limitations of our own heuristic. Previous accounts viewing different heuristics as incompatible perspectives, such as Levins' $(1998,2006)$ and Winther's (2006), seem to lack resources to argue for the above kind of pluralisms, despite what the authors suggest.

A case study of this paper is concerned with predation and the case is connected to two mega parameters discussed in the paper, namely, resources and total predation response. Other cases concerning mega parameters include competition theory and its mega parameters, such as competition coefficients, Tilman's $\mathrm{R}^{*}$, and environmental heterogeneity; selection, drift, speciation, and dispersal (Vellend 2010); the selection coefficient in population genetics and fitness (Cohen 1985); and body size in allometries and scaling laws. I have

\footnotetext{
${ }^{3}$ Some notable defenders of holistic models in ecology include MacArthur, Lotka, Volterra, May, and Levins. Some notable defenders of reductionistic models in ecology include Schoener, Tilman, and Grimm.
} 
examined some of the latter cases of mega parameters elsewhere (Raerinne 2013a; Raerinne and Baedke 2015).

\section{Holism and Sufficient Parameters}

Robustness analysis is a search for similar or convergent results of different models of a common phenomenon (Levins 1966; Weisberg and Reisman 2008; Kuorikoski et al. 2010; Raerinne 2013b). In robustness analysis, the results of a model are compared with the results of different models of the same phenomenon. If the model and its contrasts produce similar or convergent results, then we have a robust result.

One problem in ecology is that there are different causes or mechanisms $\left(\mathrm{C}_{1}, \mathrm{C}_{2}\right.$, and $\left.\mathrm{C}_{3}\right)$ at lower levels or finer scales that have similar or convergent results $\left(E_{s}\right)$ and we do not know which of them is the actual cause or mechanism of a phenomenon. An advantage of having robust results of models that model different causes with similar effects is that their robustness counts as a warrant to use unifying, abstract, and simple models of complex phenomena. In lieu of building a model for every case where different causes or mechanisms could be at work, a modeler builds a model that is simple and abstract but robust in capturing the common effects of models. One way to build such models is by constructing a sufficient parameter into a model (Levins 1966; Lane et al. 1976).

Levins $(1966,429)$ clarified sufficient parameters as follows:

The sufficient parameters may arise from the combination of results of more limited studies. In our robust theorem on niche breadth [i.e. "in an uncertain environment species will evolve broad niches and tend toward polymorphism but a certain and diverse environment leads to specializations"] we found that temporal variation, patchiness of the environment, productivity of the habitat, and mode of hunting could all have similar effects, and they did this by way of their contribution to the uncertainty of the environment. Thus uncertainty emerges as a sufficient parameter.

The sufficient parameter is a many-to-one transformation of lowerlevel phenomena. Therein lies its power and utility, but also a new source of imprecision. The many-to-one nature of "uncertainty" prevents us from going backward. If either temporal variation or patchiness or low productivity leads to uncertainty, the consequences of uncertainty alone cannot tell us whether the environment is variable or patchy or unproductive. Therefore we have lost information. [Emphasis added.]

Wimsatt (1980, 304-305) developed the idea from a similar angle:

A sufficient parameter is thus an index which, either for most purposes, or merely for the purposes at hand, captures the effect of variations in the lower level variables (usually only for certain ranges of the values of these variables) and can thus be substituted for them in the attempt to build simpler models of the upper level phenomena. It is related to the notion of a supervenient property widely discussed in the recent philosophical 
literature... except that whereas the latter involves a deductive and therefore exact relation between lower and upper level properties, the notion of a sufficient parameter is broader, involving a relation which is inexact, approximate and usually conditional. A sufficient parameter is a heuristic tool for dealing with complexity... The notion of supervenience ... may be regarded as a kind of limiting case of a sufficient parameter, but it is I would argue, a relation which is seldom is ever found in the models of the science. Sufficient parameters, however, are frequent tools of scientists. ${ }^{4}$

In the case of a holistic sufficient parameter one is thus investigating whether different lower-level or finer-scale causes $\left(\mathrm{C}_{1}, \mathrm{C}_{2}\right.$, or $\left.\mathrm{C}_{3}\right)$, have similar effects $\left(\mathrm{E}_{\mathrm{s}}\right)$. If they have similar effects, then a modeler builds a causal or explanatory proxy $\left(\mathrm{C}_{\mathrm{abs}}\right)$ that abstracts away the differences between alternative or back-up causes $\left(\mathrm{C}_{1}, \mathrm{C}_{2}\right.$, or $\left.\mathrm{C}_{3}\right)$ with similar effects $\left(\mathrm{E}_{\mathrm{s}}\right)$. This abstract sufficient parameter $\left(\mathrm{C}_{\mathrm{abs}}\right)$ with similar effects $\left(E_{s}\right)$ is then used in models and equations instead of different specific causes $\left(\mathrm{C}_{1}, \mathrm{C}_{2}\right.$, or $\left.\mathrm{C}_{3}\right)$. In other words, lower-level or finer-scale causes $\left(C_{1} \rightarrow E_{s}\right),\left(C_{2} \rightarrow E_{s}\right)$, and $\left(C_{3} \rightarrow E_{s}\right)$ are replaced with an abstract causal proxy $\left(\mathrm{C}_{\mathrm{abs}} \rightarrow \mathrm{E}_{\mathrm{s}}\right)$ in a holistic model. Sufficient parameters function as abstract or higher-level causal or explanatory surrogates for different lower-level or finerscale causes or mechanisms that have similar effects in models of a phenomenon.

One problem in using sufficient parameters is that causes have similar, but not identical, effects, and this information is lost when using the causal proxy $\left(C_{a b s} \rightarrow E_{s}\right)$. At the same time, the abstract and higher-level relationship $\left(C_{a b s} \rightarrow E_{s}\right)$ is stable and unifying in the sense that it does not matter which of the individual causes is present $\left(\mathrm{C}_{1}, \mathrm{C}_{2}\right.$, or $\left.\mathrm{C}_{3}\right)$, since a similar effect $\left(\mathrm{E}_{\mathrm{s}}\right)$ will follow in different background conditions (back-up causes), with regard to different systems (different operative causes), and so on.

An example of a sufficient parameter is environmental uncertainty in Levins' (1966) robust theorem concerning niche breadth. There are different instances of environmental uncertainty, such as temporal variation of the environment $\left(\mathrm{C}_{1}\right)$ and patchiness of the environment $\left(\mathrm{C}_{2}\right)$, which have similar effects on species, namely, species tend to evolve broad niches and tend toward polymorphism $\left(\mathrm{E}_{\mathrm{s}}\right)$. The robustness of the results of different instances of uncertainty of environment in different models allows the use of environmental uncertainty $\left(\mathrm{C}_{\mathrm{abs}}\right)$ as a sufficient parameter in an abstract model of this phenomenon. Levins (1966) establishes this with three models, which make

\footnotetext{
${ }^{4}$ It is an interesting research topic what the connection between sufficient parameters and different concepts of supervience is, e.g. what is the minimum concept of supervenience (or emergency) that is presumed by sufficient parameters? This topic needs to be investigated elsewhere, since it is beyond the scope of this paper. At the same time, this topic is less relevant to the topics of the paper than might at first appear. First, it is not constitutive determination relations, such as supervenience or emergence (for the difference and connection between the two, see Kim 2006), which are typically conceived of as synchronic relations that are important in the given context, but causal and mechanistic relations, which involve synchronic, diachronic, and intensional relations. Second, and related to the first point, sufficient parameters are not introduced or used as a metaphysical determination relation by scientists, but sufficient parameters are explanatory and predictive tools of scientists to deal with complex systems, which is Wimsatt's point in the passage quoted above (see also my discussion in section 4). Third, reference to supervenience in this context might not be helpful, since this relation is used in diverse ways in the literature and, more importantly, there are qualms about the usefulness of supervenience as a metaphysical determination relation (see Horgan 1993).
} 
different assumptions concerning species, their fitness, genetics, and so on. Since the three models have the common result referred to, this justifies using environmental uncertainty as a sufficient parameter in a holistic or general model of this phenomenon.

A model with a sufficient parameter shows how different modeled causes or mechanisms that have similar results can be presented in a unifying theoretical framework $\left(\mathrm{C}_{\mathrm{abs}} \rightarrow \mathrm{E}_{\mathrm{s}}\right)$ which abstracts away the differences between causes or mechanisms. If a result is robust, the job of identifying the actual cause or mechanism $\left(\mathrm{C}_{1}, \mathrm{C}_{2}\right.$, or $\left.\mathrm{C}_{3}\right)$ can be irrelevant for some modeling purposes.

Unification by means of sufficient parameters is a beneficial feature of model-based explanations and predictions. Unification provides us with conceptual and cognitive economy, coherence, and systematization of knowledge. However, nothing in the above presupposes that for explanations the identification of the actual cause or mechanism has become redundant, owing to the robustness of the results of models. It is here where "lies its power and utility, but also a new source of imprecision," as Levins expressed the dual nature of sufficient parameters in the passage quoted above.

The above imprecision concerning the actual causes and mechanisms of a phenomenon in using sufficient parameters is one of the main motivations behind mechanistic or reductionist models in the literature (Tilman 1977, 1980, 1990; Schoener 1986). The defenders of mechanistic models want to expose the actual causes and mechanisms behind phenomena. Another reason is that mechanistic models can sometimes be used to provide more accurate predictions and explanations than holistic models of the same phenomenon with sufficient parameters.

For a holist, a resource is valid as a sufficient parameter $\left(\mathrm{C}_{\mathrm{abs}}\right)$, because resources have similar effects on population growth rates $\left(E_{s}\right)$ : "[a] resource is a factor which, through some range of availabilities, leads to higher population growth rates as its availability is increased and which is consumed, in the broad sense, by the population" (Tilman 1977, 363). ${ }^{5}$ However, Tilman $(1980,1986)$ has shown that the abstraction can be unpacked into distinct and more detailed causal factors $\left(\mathrm{C}_{1}, \mathrm{C}_{2}, \ldots\right)$. Different resources have the similar general effect $\left(\mathrm{E}_{\mathrm{s}}\right)$ mentioned in that they have a positive effect on the population growth rates when there is a certain amount of a resource to be consumed. At the same time, different

\footnotetext{
${ }^{5}$ As noted by an anonymous referee, the above definition of resources - which was meant to apply to ecology only - was taken from Tilman. I do not see any contradiction in the fact that Tilman is not a holist, but a reductionist, and that his definition can be used as a sufficient parameter of resources by holists. In fact, Tilman needs a working definition of abstract resources if he wants to de-abstract this definition with his mechanistic taxonomy of resources (see below). Furthermore, this mechanistic taxonomy of resources, as I argue later on, can be used by holists as well to see when their abstract definition of resources as a sufficient parameter is not valid. The main thesis of this paper is that holism and reductionism are not contradictory but complementary as heuristics. If the reader is dissatisfied with this abrupt discussion of resources, as the anonymous referee was, let me motivate the discussion. First, there probably are not many other concepts in ecology that are theoretically more important than resources. Even most definitions of niche presume a definition of resources, especially the Hutchinsonian concept; and consequently, resources are a central concept in the context of competition theory as well. Second, other definitions of resources do exist in the literature, but Tilman's mechanistic taxonomy of resources is currently among the best and most workable of definitions, even though it includes missing items and perhaps even inconsistencies. Finally, I utilize Tilman's mechanistic taxonomy of resources - his de-abstraction of a sufficient parameter - in section 3 to show how an abstraction by reductionists can also founder (see the total predation response in section 3 ).
} 
resources $\left(\mathrm{C}_{1}, \mathrm{C}_{2}, \ldots\right)$ have different kinds of specific effects on population growth rates as well, which, in turn, have different effects on the outcome of interspecific competition (see below) and consumer-resource interactions (see total predation response in section 3$)$.

Tilman $(1980,1986)$ has provided us with a mechanistic taxonomy of resources, which de-abstracts the abstract sufficient parameter used by holists. What follows is my paraphrasing of Tilman's mechanistic taxonomy of resources. Readers interested in original definitions should consult Tilman's papers. Let $\left(\mathrm{C}_{1}\right)$ and $\left(\mathrm{C}_{2}\right)$ stand for two different resources. There are three main types of resources: essential, substitutable, and hemi-essential resources. Resources are essential when both $\left(\mathrm{C}_{1}\right)$ and $\left(\mathrm{C}_{2}\right)$ are required for the growth of a population, that is, $\left(\mathrm{C}_{1}\right)$ and $\left(\mathrm{C}_{2}\right)$ are necessary, but neither $\left(\mathrm{C}_{1}\right)$ nor $\left(\mathrm{C}_{2}\right)$ alone is sufficient for growth. In the case of substitutable resources, $\left(\mathrm{C}_{1}\right)$ and $\left(\mathrm{C}_{2}\right)$ are sufficient, but neither $\left(C_{1}\right)$ nor $\left(C_{2}\right)$ is necessary for growth; that is, either $\left(C_{1}\right)$ or $\left(C_{2}\right)$ is needed for growth. Finally, in the case of hemi-essential resources, $\left(\mathrm{C}_{1}\right)$ alone can be both necessary and sufficient for growth, but $\left(\mathrm{C}_{2}\right)$ can at least partly substitute for $\left(\mathrm{C}_{1}\right)$.

For the purposes of this paper, the more interesting cases are the different kinds of resources falling under the rubric of substitutable resources, namely, perfectly substitutable, complementary, antagonist, and switching resources (see below and discussion in sections 3 and 4$).\left(\mathrm{C}_{1}\right)$ and $\left(\mathrm{C}_{2}\right)$ are perfectly substitutable resources if $\left(\mathrm{C}_{1}\right)$ can be substituted for $\left(\mathrm{C}_{2}\right)$ and vice versa. $\left(\mathrm{C}_{1}\right)$ and $\left(\mathrm{C}_{2}\right)$ are complementary resources in the case of positive emergence: when $\left(\mathrm{C}_{1}\right)$ and $\left(\mathrm{C}_{2}\right)$ are consumed together, their combined effect on growth is greater than what the additive sum of $\left(C_{1}\right)$ and $\left(C_{2}\right)$ would suggest when $\left(C_{1}\right)$ and $\left(C_{2}\right)$ are not consumed together. $\left(\mathrm{C}_{1}\right)$ and $\left(\mathrm{C}_{2}\right)$ are antagonist resources in the case of negative emergence: when $\left(\mathrm{C}_{1}\right)$ and $\left(\mathrm{C}_{2}\right)$ are consumed together, their combined effect on growth is smaller than what the additive sum of $\left(\mathrm{C}_{1}\right)$ and $\left(\mathrm{C}_{2}\right)$ would suggest when $\left(\mathrm{C}_{1}\right)$ and $\left(\mathrm{C}_{2}\right)$ are not consumed together. $\left(\mathrm{C}_{1}\right)$ and $\left(\mathrm{C}_{2}\right)$ are switching resources when organisms can switch between $\left(C_{1}\right)$ and $\left(C_{2}\right)$ when $\left(C_{1}\right)$ or $\left(C_{2}\right)$ has a more positive effect on the growth. When providing more accurate and specific predictions, the differences between the mentioned resources matter, for instance, in the context of competition between species for limited resources.

In a homogeneous environment, a necessary condition for the stable coexistence of two species with similar niches competing for two essential limiting resources is that each species consumes relatively more of the resource that is most limiting to its own growth, i.e. species should be competitively dominant vis-à-vis the resource that is the most limiting to the species. However, this is not a necessary condition for the coexistence of competitor species for other types of resources, such as substitutable or hemi-essential resources (Tilman 1980). In a spatially heterogeneous environment, there is no simple limit to the number of coexisting species competing for two (hemi-)essential resources, whereas the number of coexisting species competing for switching or antagonist resources cannot exceed the number of their limiting resources (Tilman 1986). We thus get more system-specific, accurate, and testable predictions and explanations from models when the abstraction, such as resource $\left(\mathrm{C}_{\mathrm{abs}}\right)$, is de-abstracted or reified into distinct and detailed causal factors $\left(\mathrm{C}_{1}, \mathrm{C}_{2}\right.$, or $\left.\mathrm{C}_{3}\right)$.

However, the moral is not that holists are sloppier in explanations (or predictions) than reductionists or mechanists. For a holist, similarity rather than identity of effects of different causes is enough to justify the abstraction, because they aim at general and unifying explanations, whereas mechanistic modelers aim 
at more fine-grained or precise explanations that are system specific. The difference is between favoring different kinds of dimensions of explanatory power (see section 4).

Mechanistic models thus have advantages over holistic models in being able to produce more exact and testable explanations and predictions. The other side of the coin is that mechanistic models lose generalizability in that the class of phenomena with which they are concerned may be limited, i.e. they become case studies of narrow scope. Moreover, when modeling a phenomena with lower-level causal components or finer-scale details, the models tend to become complex, intractable, involve many functional and interacting components with a large number of parameters, are hard to comprehend, and so on. To avoid these pitfalls, reductionists make use of abstractions as well, which I call aggregative parameters.

\section{Reductionism and Aggregative Parameters}

In aggregation, one integrates or merges different lower-level or finer-scale causal components with different or similar effects to extrapolate what their combined effect would be. To do so, a modeler forms a parameter, which combines the effects of different causal components into a single monadic coarse scale component, the aggregative parameter, which involves abstracting away the details of the organization of the system. In other words, with an aggregative parameter one builds a composite abstraction of different causal components $\left(\mathrm{C}_{\mathrm{i}}\right)$ with different or similar effects $\left(\mathrm{E}_{\mathrm{i}}\right)$, using a function that combines the effects of different components together into one causal proxy $\left(\mathrm{C}_{1+n}\right)$ which has a compound effect $\left(E_{1+n}\right)$. An aggregative and abstract function or relation $\left(C_{1+n} \rightarrow E_{1+n}\right)$ is then used in mechanistic models. Given that different components or forces are typically responsible for the system-level properties in ecology, many would welcome a synthesis of ecology by means of aggregative parameters (cf. Schoener 1986 and Vellend 2010).

There are general problems with aggregation. Different individual causal components $\left(\mathrm{C}_{\mathrm{i}}\right)$, might fail to be operative in different background conditions. Thus, their combination $\left(\mathrm{C}_{1+n}\right)$ is not stable or projectable if the background conditions change. Addition of components and their subtraction typically have the result that a qualitatively different compound effect than $\left(E_{1+n}\right)$ is produced. The organization of how components are put together affects the system behavior. That is, the organization of components matter, and changes in organization can produce emergent effects. In general, rearrange, substitute, add, or subtract some of the individual components $\left(\mathrm{C}_{\mathrm{i}}\right)$ and the compound effect $\left(\mathrm{E}_{1+\mathrm{n}}\right)$ might be qualitatively different (for more detailed cases, see below and Wimsatt 1986).

There have been heated debates as to what kind of heuristics ecological modelers should favor. These debates are related to the issue of what kinds of abstractions ecologists should use in their models. In his defense of mechanistic or reductionistic ecological models over and above traditional holistic models, Schoener suggests that a strong form of mechanistic reductionism holds in ecology; namely, that the higher-level properties of ecological systems are aggregates of their components' properties in isolation: 
The phenomena of interest in population ecology are aggregate properties of the individuals composing the population; examples are age structure, sex ratios, growth rates, and reproductive schedules. Community ecology deals with a group of populations in some place. Here the aggregate properties of interest concern the various species populations: abundance distributions, species diversity, species-turnover rates, and so on. (Schoener 1986, 91.)

As a general or unqualified thesis, Schoener's claim that the properties of ecological systems are aggregates of their components' properties in isolation is implausible. In fact, as a general empirical claim it is false. Consider predation, which is a causal factor that has an effect on prey mortality and population density - a positive causal mortality factor of prey and thus a negative (regulatory) causal factor of prey's population density. Predation is an abstraction of the components of predator-prey systems. ${ }^{6}$

Holling (1959) is a classical study on predation focusing not only on the components of predation, but on how the components make up and interact to produce a total predation response. The total predation response expresses the total proportion of mortality rate of prey by its predators, determining whether the

\footnotetext{
${ }^{6}$ How can I claim that predation is both a causal factor and an abstraction? I thank an anonymous referee for pointing out this issue to me. As has been argued by Reisman and Forber (2005), higher-level abstract variables can be regarded as causes in the sense of being difference-makers to their effects. Even though their arguments concern natural selection and drift as abstract population-level causes, the arguments can be applied to predation. In fact, the basic idea behind Holling's (1959) predation study (see below) is to investigate how the total predation response, as a higher- or population-level cause, affects the densities of prey populations, what are the components of this abstract cause, and how the components interact to produce the populationlevel cause. Another classical study in this context is Paine (1966). Paine was interested in how observed high local diversities of many communities are compatible with the competitive exclusion principle rather than being exceptions to it. His study and other studies that followed afterwards by him and others manipulated predation and investigated what effects different levels of predation - as a population- or community-level cause - has on the diversity of communities and population densities of competitor species in communities. Paine and others discovered experimentally that removing one or more of the top predator species from their communities had the effect of reducing the alpha diversity of communities' consumer species: without a common predator species, a few (and perhaps even one) competitively dominant consumer species come to monopolize the community by outcompeting other consumer species. Paine's explanation was that predators can mediate the coexistence of their prey and maintain the local diversity of a community at a high level by keeping competing prey populations' densities or abundances below a level at which the competition would become so severe as to cause local extinctions of species from the community. The story about predation as an abstract cause does not end here, but gets even more abstract. These and similar experimental findings and explanations of exceptions to the competitive exclusion principle were later generalized as the intermediate disturbance rule. According to this rule, intermediate levels of abiotic or biotic disturbances, such as predation, pathogens, aridity, storms, and fires are capable of mediating the coexistence of competitor species and thus maintaining the local diversity of a community at a relatively high level. Different instances of intermediate disturbances have the same or a similar effect, for instance, through reduction of population densities of competitor species, which counter the strong competitive effects between species. (Too small or too infrequent disturbances lead to local extinctions of competitively inferior species by competitively dominant ones, whereas too intense or too frequent disturbances allow for the few species that are the most stress-tolerant to exclude other species from a community.) Note that, in the above rule, "intermediate disturbance" works as an abstract causal factor, a sufficient parameter, of which predation is one instance. In sum, abstract causes aggregative and sufficient parameters - can be treated and are treated by ecologists as causes in the sense of being difference-makers to their effects.
} 
density of prey population is regulated by its predators. The shape of the total predation response curve helps to determine whether the predators are able to control their prey population density. Expressed in the terminology of this paper, Holling's idea was to study whether the total predation response is an aggregative property of its components' properties in isolation and how the total predation response, as a biotic density-dependent factor, regulates the density of prey species populations.

Holling's study originally started as a field investigation of a simple predator-prey system in a uniform and homogenous environment, which was causally isolated from abiotic and biotic background conditions that could interfere with the system. The ingenuity of Holling's study was that he stripped down this simple predator-prey system into its basic components to see how the components produced the supposedly aggregate property of the system, namely, the total predation response. Later, he started to investigate the basic components of predation in the laboratory using other systems. His investigations showed that the total predation response is more than the sum of the properties of predatorprey systems' components' properties in isolation, i.e. an emergent rather than a simple aggregate property. The results of his investigations apply to predator-prey systems in general. In fact, later studies on predation have elucidated different ways in which the total predation response is not an aggregative property of predator-prey systems in general. I will paraphrase the results of Holling's study using the terminology of this paper.

Holling's (1959) study was originally concerned with the predation of cocoons of a sawfly species by three mammal species. Due to the simplicity of the predator-prey system and the uniformity of the background conditions, there initially seemed to be two main additive components to the total predation response: 1) how predators respond dietarily to changes in their prey density, such as by consuming their prey in fewer or greater numbers (the so-called functional response of predators) and 2) how predator density is affected by the density of their prey (the so-called numerical response of predators). A functional response $\left(\mathrm{C}_{1}\right)$ is the change in predator's consumption as a function of change in prey density, whereas a numerical response $\left(\mathrm{C}_{2}\right)$ is the change in predator density as a function of change in prey density. The total predation response is a combination of functional and numerical responses. Were the total predation response an aggregative property of predator-prey systems, then the effects $\left(E_{1}\right)$ and $\left(E_{2}\right)$ of predators' numerical $\left(\mathrm{C}_{1}\right)$ and functional $\left(\mathrm{C}_{2}\right)$ responses on the mortality of their prey could be combined as independent (additive) elements, and the aggregative function $\left(\mathrm{C}_{1+2} \rightarrow \mathrm{E}_{1+2}\right)$ could be applied to predator-prey systems to model total predation responses.

However, as Holling notices, prey density has different kinds of effects on the functional response of predators. There is no typical or general response, although some qualitative general results can be stated, cf. the classical type I, II, and III functional response curves in Box 1. The numerical response is more varied, and there are species that show no numerical response, whereas other species show a marked positive or even negative ${ }^{7}$ numerical response vis-à-vis changes in the density of their prey. The total predation response is thus a

7 Tilman's mechanistic or reductionistic taxonomy of resources contains missing items (see section 2). An example is resources that under certain conditions - as individual causal factors - can have a negative effect on the population growth rates, a case which should not be confused with antagonist resources. 
combination of two possible continua: "a negative numerical response to a marked positive numerical response" and "no functional response to a marked positive effect in functional response." Thus, there is no general or universal total predation response, because the components vary and/or are taxon or system specific. $^{8}$

Consider a simple case where there is no numerical response in predators, but predators respond only functionally to changes in the density of their prey; predator species with longer generation times than their prey fit this assumption at least approximately. In this case, the total predation response curve is determined by the functional response curve of predators (see Fig. 2 in Box 1), and consequently the prey mortality rate is determined by the functional response of predators. With a linear, type I functional response curve, the mortality rate of prey is constant and the prey population density is regulated by its predator, which is thought to lead to classical Lotka-Volterra population dynamics in the predatorprey system, where there are cyclic and coupled changes in both predator and prey population densities. ${ }^{9}$ With a rectangular hyperbola, type II functional response curve, the prey mortality rate declines with increases in prey density: predators are able to regulate their prey population density at low prey densities, but the death rate of prey due to predation starts to diminish at moderate prey densities, and the prey population is even capable of escaping the checks of its "natural enemies" at high population densities. With an S-shaped, type III functional response curve, the mortality rate of prey due to predation is low at its low density, then it starts to accelerate at moderate prey densities, because the functional response of predators accelerates with the changes in their prey density, and finally the mortality rate of prey starts to diminish and even plateaus at high prey density, showing that there is little or no regulation of prey population density by its predators at low and high prey density, even though the prey population density may be regulated by predators at moderate prey population densities.

What is more, the total predation response is typically affected by many other components of the predator-prey system and its background conditions than functional and numerical responses, which can have various and even opposing effects on total predation, on the two mentioned basic components, on the interactions between the components, and so on. As examples of additional

\footnotetext{
${ }^{8}$ The above does not yet necessarily establish that the total predation response is not an aggregative property. This is because the total predation response could be an aggregative property of a system's specific functional and numerical responses, even though the details of aggregation vary from one system to another. In a certain predator-prey system, the total predation response could be an aggregative property of a numerical response curve 1 in Figure 1 and a functional response curve 4 in Figure 2. In this case, the total predation response curve would have the form of a curve 5 in Figure 3 . In another system, the total predation curve could be an aggregative property of a numerical response curve 2 in Figure 1, and a functional response curve 4 in Figure 2. In the latter case, the total predation response curve would have the form of a curve 6 in Figure

3 . In both cases, the total predation response would thus be an aggregative property of each system's components' properties in isolation. This would be an empty victory for reductionists, however. Aggregations would lack generality and extrapolability.

${ }^{9}$ There is an ongoing debate whether different versions of Lotka-Volterra predator-prey models produce robust results (e.g. Weisberg and Reisman 2008). The main issue is whether the results of Lotka-Volterra models are affected by the simplifying assumptions used in the models. This debate has its own merits. However, if the core assumption of this model itself - the idea that the functional response curve of predators is or approximates type I curve - is a bad representation of most predator-prey systems (and it often is), robustness analyses of these models cannot establish that they are good models.
} 
components, consider the availability and quality of alternative resources for predators, the degree of resource specialization of predators, immigration and emigration of predators and prey, the traits of prey species, such as their palatability or defense mechanisms, and competition between predator species for resources (Holling 1959, 305-308; Korpimäki and Norrhdahl 1989; and Persson and Diehl 1990). To have a total predation response, the effects of the additional components need to be considered on the already existing components and/or added to the total predation response. The effects that these other components have on the already mentioned two response functions need not be uniform, nor are these other components uniform in effects in different background conditions, taxa, community structures, etc. That is, the total predation response is not an aggregative property of predator-prey systems in general.

The nature of alternative resources matter. Alternative perfectly substitutable resources for predators can negatively affect their functional response, but positively affect their numerical response, whereas antagonistic resources can have negative effects on both functional and numerical responses. Essential, complementary, and switching types of alternative resources have different kinds of effects - positive, negative, or none - on the numerical and/or functional response of predators. The additional components are thus capable of interacting in qualitatively different ways with the basic components and/or are capable of having qualitatively different effects on the total predation response, the way the components interact with each other, and so on.

As predation increases, prey can respond to this in various ways, such as producing chemicals that make the prey less palatable or organizing to defend themselves. This can have a negative effect on the functional response of predators, it can have an effect on the way in which alternative resources are consumed by predators, which in turn can have negative or positive effects on the numerical or functional response of predators. And there are higher-order interactions between different components that need to be considered, such as the effects of competition between predator species for their prey, the predators of predators, which are capable of producing positive and negative causal feed-back loops affecting the system and its components in various and qualitatively diverse ways.

The upshot is that the total predation response is typically not an aggregative property of its components' properties in isolation, but an emergent property of a predator-prey system. The way the components interact, how they are organized, what are the biotic and abiotic background conditions, and so on, do matter and produce qualitative different or emergent total predation response curves (see Box 1). Total predation is mechanistically explainable, but it is more than the sum of its components' properties in isolation. Other cases of nonaggregate properties include, for instance, fitness (Cohen 1985), Tilman's complementary and antagonistic resources (see section 2), and Vellend's (2010) selection, drift, speciation, and dispersal. Many and perhaps even most of the higher-level properties of systems in ecology cannot be treated or modeled as mere aggregates of their components' properties in isolation, in contrast to what seems to be suggested by Schoener (1986) in the passage quoted above.

What was said about problems of treating the properties of ecological systems as aggregates was not aimed to show that aggregation is futile. Nor was the above presented as an argument against reductionism as a heuristic.

Aggregation is an effective method to make complex mechanistic or reductionist 
models simpler, more mathematically or analytically tractable, less sensitive to parametric variation in results, less prone to the parameter value estimation problems, and so on (Levin 1991, 1992). Aggregation is an effective strategy for abstracting with regard to simple systems and their properties in homogenous or constant environments. Approximations and partial aggregations sometimes suffice (Iwasa et al. 1987; Iwasa et al. 1989). Aggregation errors can be corrected and different methods are discussed in the literature (Gardner et al. 1982; Rastetter et al. 1992). Carelessly treating or naively assuming, however, in the sense of Schoener (1986) and perhaps Vellend (2010), that the higher-level properties of ecological systems are mere aggregates of their components' properties in isolation can lead to simplification errors and biases in results, non-projectable results, and unjustified policy- and decision-making recommendations.

Finally, the above discussion in this and the previous section on two kinds of abstractions utilized by holists and reductionists shows that mechanists are not committed to the unqualified thesis that adding details, i.e. de-abstracting, always or typically improves their explanations (see discussion in Machamer et al. 2000, 15-18; Kaplan 2011, 347-348; Levy and Bechtel 2013; Boone and Piccini 2016, 1517-1519; and Miłkowski 2016 on this topic). Mechanists use abstractions to make better model-based explanations and predictions, it is just that the abstractions utilized are different from those used by holists. Both adding certain kinds of details, such as de-abstracting a sufficient parameter and omitting certain other kinds of details, such as utilizing an abstract aggregative parameter, can improve a mechanistic model without necessarily jeopardizing the basic tenet of mechanistic explanations being system-specific, constitutive, and precise. 
Fig. 1. Numerical response curves: predator density (y axis) vs. prey density (x axis)

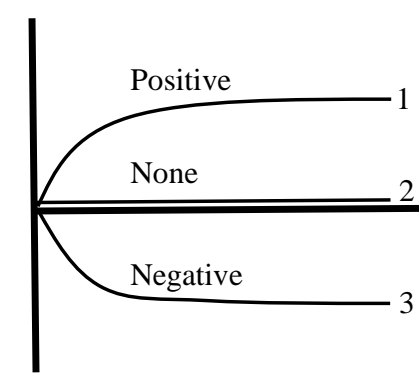

Fig. 2. Functional response curves: predator consumption (y axis) vs. prey density ( $\mathrm{x}$ axis)
Fig. 3. Total predation response curves: total predation (y axis) vs. prey density ( $\mathrm{x}$ axis)

Box 1. Numerical, functional, and total predation curves (partly adopted from Holling 1959).

Fig. 1. Curve 1: a positive numerical response. Curve 2: no numerical response. Curve 3: a negative numerical response. Fig. 2. Curve 4: a positive functional response, the so-called Type II functional response, which has the form of a rectangular hyperbola. Type I and III curves are represented as dashed curves. Fig. 3. Curve 5: the total predation response: curves 1+4. Curve 6: the total predation response: curves $2+4$. Curve $7:$ the total predation response: curves $3+4$. Even in the simple case presented here, where there is only one type of functional response, Type II, which is added to three different numerical response curves, shows that there is no general total predation response curve, but quantitatively (curves 5 and 6) and qualitatively (curve 7) different kinds of curves do exist. The case is more complex when one adds different functional response curves to three basic numerical response curves (dashed curves in Fig. 2, which represent the so-called Type I (linear) and Type III (S shaped) functional curves): qualitatively very different kinds of total predation response curves than 5, 6, and 7 follow. Note that the total predation response is presented in Fig. 3 as an additive or aggregative of numerical and functional response curves. Actually, this is not necessarily the case, but numerical and functional response can interact in emergent ways. However, let us suppose that the total predation response is additive of the two components. Now, add the effects of other components of total predation, such as availability of alternative resources, to the functional or numerical response component and/or to total predation. These can affect both numerical and functional response curves in various and even opposite ways. For instance, numerical responses can be made more positive or negative, functional responses can become more positively pronounced, and so on. The effect that alternative resources have on total predation response curves can be very varied, and qualitatively very different curves than curves 5-7 follow. Similarly, competition between predators for resources can have different effects on the numerical or functional response or on the total predation response, and so on. That is, there are direct and indirect interactions between the components of the total predation response curve that have varied and emergent effects on the form of the total predation response, on the components of the curve, how the components interact, and so on - cf. the dashed total predation response curve in Fig. 3, which presents a hypothetical curve of how different components could "add up" to form a complex and non-aggregative total predation response curve. 


\section{Model Pluralism and Complementarity of Heuristics}

In ecology, the strategy of using a diverse set of holistic and reductionistic models to gain understanding of a common phenomenon, such as competition or predation, is typical (Levins 1966; Levin 1991, 1992). This diversity of models for one and the same phenomenon cries out for an explanation. Is the diversity of models a good or a bad thing? Should it be eliminated or maintained? And for what methodological, epistemic, or pragmatic reasons?

Pluralism implies diversity, but diversity does not necessarily imply pluralism. The accounts viewing different heuristics as contrary or contradictory perspectives (Levins 1998, 2006; Winther 2006) seem to imply that there is no model pluralism, but a diversity of incompatible models for a common phenomenon. If holism and reductionism are contradictory, there is no pluralism. This is because the truth of holism implies that reductionism is false about their common phenomenon and vice versa. Similarly, if holism and reductionism are contrary, there is no pluralism, because the two do not share a phenomenon of which both can be at least partially true. Thus, whether model diversity in ecology implies model pluralism - and whether it is a vice or virtue - remains an unresolved issue. ${ }^{10}$

In this section, I argue that model diversity implies beneficial model pluralism. Different methodological functions, strengths, and limitations in using sufficient parameters and aggregative parameters were discussed in previous sections. Here, I present an epistemic defense of model pluralism by arguing for the explanatory complementarity of reductionism and holism as heuristics. Different models utilizing different heuristics have different epistemic strengths and limitations and they provide incomplete but complementary research perspectives of cognitively limited beings.

Let me make a distinction between two kinds of causal explanations, simple causal claims and mechanistic explanations (Raerinne 2011). A simple causal claim describes a causal relationship between the phenomenon-to-be-explained and the thing that does the explaining. It refers to a causal explanation in which one has a causal relationship between variables, but no or little account - or mechanistic explanation - as to why or how the relationship holds between the variables. A simple causal claim is thus a manipulable black box relation holding between variables.

Describing a mechanism of a phenomenon is not contrary to describing what the causal relationship of a simple causal claim of that phenomenon is. Instead, a mechanistic explanation is a complement to a simple causal claim, since

\footnotetext{
${ }^{10}$ A trivial argument for the diversity of models exists. A similar argument is utilized by many who confuse diversity with pluralism (e.g. Beatty 1995, 65-75 and Jamniczky 2005). If different models applied to the same general phenomenon, but to different systems, so that holistic models are used to investigate complex systems of a phenomenon, whereas reductionistic models are used to investigate simpler systems of this phenomenon (or vice versa), this would account for the diversity of models. At the same time, this would not justify pluralism, since there is no pluralism about models that do not share a target. Instead of pluralism, there would be a diversity of local monisms. Alternatively, one could call this case global pluralism in the sense that at the discipline or science level different heuristics are needed to account for the whole diversity of its target systems. Instead of applying this argument, I try to provide arguments in support of epistemic and methodological pluralism in theoretical ecology, which does not just establish model diversity, but instead the local pluralism of holistic and reductionistic models.
} 
it describes how the causal relationship produces its phenomenon-to-be-explained. A mechanistic explanation describes the underlying mechanism within the system by showing how the system is constituted and how this produces the phenomenon-to-be-explained. The suggestion is to view traditional holistic ecological models, such as the Lotka-Volterra competition and predation models utilizing abstract resources or competition coefficients and Levins' (1966) environmental uncertainty model as providing simple causal claims, whereas reductionistic models provide us with mechanistic explanations of the former.

Holistic and mechanistic models are not exclusive explanatory rivals in the sense that when one is true, then the other must be false. Rather than being contrary or contradictory, they can be viewed as complementary explanations.

Traditional holistic models investigate what would happen to community- or population-dependent variables if community or population independent variables were manipulated. These are simple causal claims in the sense that we investigate what would happen to a system if some of its higher-level variables were manipulated without worrying about what is the specific population or individual level causes(s) or mechanism(s) behind the change in the system. Mechanistic models investigate how this same change happens by accounting for the change in dependent variables in terms of lower-level - population- or individual-level independent variables (Tilman 1977, 1980, 1990; Schoener 1986; Grimm 1999). Mechanistic models investigate what is the specific mechanism responsible for this or that change in variables in which community models are derived from population models and population models from individual-based models.

When one has a mechanistic account of how and why a higher-level simple causal relationship holds, this does not show that the higher-level relationship becomes non-explanatory or redundant. In fact, both holistic and mechanistic models have become more reliable in the sense that we have a holistic model with simple causal claims which is convergent with a possible modeled mechanism at a lower level. Sometimes the models provide non-convergent results with regard to certain parameter or variables values. It is not a priori correct to blame holistic models for the failure. In fact, the specific simplifying assumptions about the mechanism, such as the aggregative assumptions used, and even the core assumptions concerning the mechanism might be incorrect. In the case of nonconvergent results, it may be the case that holistic and mechanistic models need to be modified, simplifying assumptions need to relaxed, the results of models need to be restricted to certain parameter or variable values, systems, and so on.

The usual reply is that mechanistic models give better or deeper explanations than holistic models of the same phenomenon which are based on simple causal claims. ${ }^{11}$ The explanatory power of an explanation is not, however, a one-dimensional attribute. Ylikoski and Kuorikoski (2010) have distinguished between five different dimensions of explanatory power of an explanation: nonsensitivity (stability of an explanatory relationship with regard to changes in background conditions), precision (how well the explanation characterizes the

${ }^{11}$ An anonymous referee asked exactly whose reply this is. However, the relevant question is where and when the claim is true. Compare the specific predictions and explanations given by individual-based mechanistic rabies models (Jeltsch et al. 1997; Thulke et al. 1999) to classical holistic reaction-diffusion rabies models (Murray et al. 1986). Individual-based rabies models give more exact, accurate, and testable predictions and better explanations of rabies epizootics than classical holistic models. In this context, the authors thus have a justification to claim that mechanistic models give better explanations than holistic reaction-diffusion models. 
object of explanation), factual accuracy (lack or innocence of simplifying assumptions used in explanations), unification, and cognitive salience (ergonomics of explanation - how easily the explanation can be followed and understood by limited cognitive beings). There are trade-offs between the different dimensions of explanatory power. More importantly, holist and mechanistic models have different strengths and limitations with regard to different dimensions of explanatory power.

Mechanistic models usually give more precise explanations than holistic ones, because they include details of causal components and mechanisms rather than sufficient parameters (see discussion of resources in section 2 and footnote 11). Increased precision and complexity in models usually comes at the cost of decreased analytical or mathematical tractability. Moreover, complex models are more difficult to understand than simpler models, thus, their cognitive salience is typically diminished. An explanation characterized too precisely can make it too complex for us to understand it (cf. Putnam 1975; Nathan 2012; Raerinne and Baedke 2015; Raerinne forthcoming). In other words, precision reduces cognitive salience. This is a crucial problem for sciences dealing with complex phenomena, such as ecology.

Holistic models are more unified than mechanistic models. In a holistic model with a sufficient parameter, knowing the actual cause or mechanism could be irrelevant because the causal relationship holds regardless of which of the cause(s) or mechanism(s) were operative. They are also more cognitively salient, because they are less precise and include sufficient parameters. In general, there is a trade-off between cognitive salience and precision as dimensions of explanatory power.

Holistic models are often more stable or less sensitive than mechanistic models in the sense that they utilize higher-level causal proxies or sufficient parameters and omit mechanistic details. If a cause does not operate when certain background conditions are in place, or if it does not operate in a certain system/taxon, then another alternative or back-up cause with similar effects might be operative and the model applies to the situation (see environmental uncertainty in section 2). Thus, an explanation utilizing sufficient parameters can be claimed to be a stable or an insensitive one.

The proponents of mechanistic models sometimes claim that their models include fewer, better, or less factually inaccurate simplifying assumptions than holistic models. Both holistic and mechanistic models include simplifying assumptions. At the same time, the simplifying assumptions are of different kind, and there is probably no general metric to evaluate different kinds of simplifying assumptions associated with different heuristics as to how good, reliable, or innocent they are. Sufficient and aggregative parameters as abstractions have different methodological functions, strengths, and sources of errors. Neither is in any straightforward sense less innocent or better than another as a simplifying assumption. Thus, insofar as factual accuracy is concerned, it seems to be a comparable attribute of explanations within a heuristic, not between heuristics.

Thus, holistic and mechanistic models are not contrary as explanations. If they provide us with convergent results, then they can be viewed as complementary. Moreover, given the many and some conflicting dimensions of explanatory depth or power, it is not self-evident that mechanistic models provide us with better explanations than holistic models. Reductionists and holists focus on the same phenomenon, of which both can be true as explanations. But they 
prefer different dimensions of explanatory power: reductionists want their explanations to be precise and system specific, whereas holists favor explanations that are unified, cognitive salient, and non-sensitive.

Several authors have previously discussed different dimensions of explanatory power (cf. Fodor 1974; Putnam 1975; Kitcher 1984; Weslake 2010; Haug 2011). In contrast to the account of explanatory power presented above, these accounts are one-dimensional, for instance, referring to the greater generality of higher-level explanantia or mechanistic details of lower-level explanantia as the dimension conferring the explanantia with a high explanatory power. The multi-dimensional account of explanatory power not only can accommodate the idea of the one -dimensional accounts via, for instance, unification or precision, but the one-dimensional accounts lack the flexibility and diversity of the multi-dimensional account. Finally, the multi-dimensional account is biased neither towards reductionism nor holism, as the one-dimensional accounts are, but can be used to analyze explanatory strengths and limitations of both kinds of explanations. Thus, the multi-dimensional account of explanatory power is more comprehensive than and superior to the one-dimensional accounts.

In addition to the above epistemic argument for the complementarity of heuristics, a methodological argument for their complementarity exists. This applies even if mechanistic and holistic models were not true of a common phenomenon as explanations. Holism and reductionism are research perspectives on nature having their distinctive methodological strengths and limitations, which can be revised, criticized, and corrected. And we often need another heuristic to see and correct for the limitations of our heuristic, as the case of a mechanistic taxonomy of resources illustrates.

Tilman's $(1980,1986)$ de-abstraction of resources (discussed in section 2) in part helped to spell out more accurately under what conditions resources can or cannot be successfully treated as a holistic sufficient parameter. Note that this is not a case of a victory for reductionism. Rather, it is a case where the perspective of one heuristic was needed to better the perspective of another. It is not only that resources cannot always be treated as a holistic abstraction, because in some research contexts the causal details of different resources matter. In fact, and contrary to the holistic abstraction of resources, it is not even true that all the resources have a positive, let alone similar, effects on the growth rates of populations, since some resources are capable of having negative effects on the growth rates of populations, as was discussed in section 3 . Thus, a reductionistic heuristic helped to see and overcome the limitations of holistic models utilizing abstract resources. However, it is not true that systems in general can be treated as mere aggregates of their components' properties in isolation, as the case of predation in section 3 demonstrated. Rather, reductionists need to adopt a more holistic perspective in modeling systems, since the organization of systems matter and cannot always be abstracted away. Finally, even the mechanistic taxonomy of resources by Tilman $(1980,1986)$ is not strictly speaking reductionistic, since it includes emergent, holistic elements, such as complementary and antagonistic resources. To provide a comprehensive and utilizable mechanistic taxonomy of resources, one thus needs to adopt a more holistic perspective in overcoming the limitations of applying a strict reductionistic heuristic to this case.

Levins (1966) argued for using a family of different models when studying a common phenomenon, where the models differ in generality, precision, and realism. In this section, I have defended the idea of model pluralism in ecology in 
explanatory and methodological terms. In fact, as I argued above, Levins' (1998, 2006) ideas concerning heuristics cannot be used to argue for model pluralism, since they imply a diversity of incompatible models.

\section{Conclusions}

I have argued that holism and reductionism as heuristics and their associated abstractions have distinctive methodological and epistemic strengths and limitations. Rather than arguing that different research perspectives are incompatible, we should view them as complementary - epistemically and methodologically. This is easy if heuristics provide us with convergent results. But when they produce non-convergent results, we should remember that all heuristics have limitations. They are research perspectives that can be corrected, revised, and criticized, and we often need another perspective to see the limitation of our own perspective.

We should aim at an ecological theory that is pluralistic in methods and explanations. In principle, I have nothing against the possibility of having a synthesis of different perspectives, revealing the true or correct nature of nature (sensu Levins 1998, 2006 and Winther 2006), even though I have disagreed that the perspectives are always or typically contrary or contradictory. Whether such a synthesis is forthcoming and whether its development and understanding is possible for cognitively limited beings, such as us, is beyond the scope of this paper. But insofar as part of the job of philosophy of science is to help scientists in their current methodological, epistemic, and evidential matters, we cannot wait for such a synthesis to occur. Instead, we have work with and accept more limited heuristics. Though heuristics have their limitations, we can effectively use them if we are aware of these limitations.

I do not suggest that the presented taxonomy of abstractions is exhaustive. Levy and Bechtel (2013), for instance, discuss a putative distinct variety of abstraction - causal connectivity - which they argue is compatible with mechanistic explanations. ${ }^{12}$ Rather, the aim was to provide a starting point for building a more comprehensive functional taxonomy of abstractions and to discuss the implications this has, for instance, for the debate whether - and/or to what extent - mechanistic accounts of explanation are compatible with the use of abstraction, that is, whether mechanistic accounts are committed to the thesis that de-abstracting or adding more details to an explanation always or typically makes the explanation better.

Acknowledgements: The author is grateful to the three anonymous referees for the journal, Miles MacLeod, Tero Ijäs, Manuela Fernández Pinto, Luis Mireles Flores, Caterina Marchionni, Alkistis Elliott-Graves, Petri Turunen, Rami Koskinen, and Alain Marciano, who provided critical and constructive comments

\footnotetext{
${ }^{12}$ It is debatable whether Levy and Bechtel (2013) has a pure case of an abstraction in mind. Their causal connectivity seems to be a hybrid case of an abstraction combining both aggregative and sufficient parameters, since it omits details of both causes with similar effects and the causal organization of a system. This is, however, a topic for another paper. There is nothing wrong with hybrid abstractions. Different target systems may require different kinds and degrees of abstractions. The pureness of a taxonomy and the effective utilization of abstractions are separate things.
} 
on previous drafts of this paper. The work was supported financially by the Centre of Excellence in the Philosophy of Social Sciences (Academy of Finland).

\section{References}

Beatty, John. 1995. The evolutionary contingency thesis. In Concepts, theories, and rationality in the biological sciences, eds. Wolters, Gereon and James Lennox, 45-81. Konstanz: Universitätsverlag Konstanz.

Boone, Worth and Gualtiero Piccini. 2016. The cognitive neuroscience revolution. Synthese 193: 1509-1534.

Cohen, Joel. 1985. Can fitness be aggregated? American Naturalist 125: 716-729.

Fodor, Jerry. 1974. Special sciences (or: the disunity of science as a working hypothesis). Synthese 28: 97-115.

Gardner, R., W. Cale, and R. O’Neill. 1982. Robust analysis of aggregation error. Ecology 63: 1771-1779.

Grimm, Volker. 1999. Ten years of individual-based modelling in ecology: What have we learned and what could we learn in the future? Ecological Modelling 115: 129-148.

Haug, Matthew. 2011. Abstraction and explanatory relevance; or, why do the special sciences exist? Philosophy of Science 78: 1143-1155.

Holling, C. 1959. The components of predation as revealed by a study of smallmammal predation of the European pine sawfly. Canadian Etymologist 91: 293-320.

Horgan, Terence. 1993. From supervenience to superdupervenience: Meeting the demands of a material world. Mind 102: 555-586.

Iwasa, Yoh, Viggo Andreasen, and Simon Levin. 1987. Aggregation in model ecosystems I. Perfect aggregation. Ecological Modelling 37: 287-302.

Iwasa, Yoh, Simon Levin, and Viggo Andreasen. 1989. Aggregation in model ecosystems II. Approximate aggregation. Journal of Mathematics Applied in Medicine \& Biology 6: 1-23.

Jamniczky, Heather. 2005. Biological pluralism and homology. Philosophy of Science 72: 687-698.

Jeltsch, F., M. Müller, V. Grimm, C. Wissel, and R. Brandl. 1997. Pattern formation triggered by rare events: Lessons from the spatial spread of rabies. Proceeding of the Royal Society of London. Series B: Biological Sciences 264: 495-503.

Kaplan, Michael. 2011. Explanation and description in computational neuroscience. Synthese 183: 339-373.

Kim, Jaegwon. 2006. Emergence: Core ideas and issues. Synthese 151: 547-559.

Kitcher, Philip. 1984. 1953 and all that. A tale of two sciences. Philosophical Review 93: 335-373.

Korpimäki, Erkki and Kai Norrdahl. 1989. Predation of Tengmalm's owls: Numerical responses, functional responses and dampening impact on population fluctuations of microtines. Oikos 54: 154-164.

Kuorikoski, Jaakko, Aki Lehtinen, and Caterina Marchionni. 2010. Economic modelling as robustness analysis. British Journal for Philosophy of Science 61: 541-567.

Lane, Patricia, George Lauff, and Richard Levins. 1976. The feasibility of using a holistic approach in ecosystem analysis. In Ecosystem analysis and 
prediction: Proceedings of a SIAM-SIMS conference held at Alta, Utah, July 1-5, 1974, ed. Levin, Simon, 111-128. Philadelphia: Society for Industrial and Applied Mathematics.

Levin, Simon. 1991. The problem of relevant detail. In Differential equations in biology, epidemiology and ecology, eds. Busenberg, Stravros and Mario Martelli, 9-15. New York: Springer.

Levin, Simon. 1992. The problem of pattern and scale in ecology. Ecology 73 : 1943-1967.

Levins, Richard. 1966. The strategy of model building in population biology. American Scientists 54: 421-431.

Levins, Richard. 1998. The internal and external in explanatory theories. Science as Culture 7: 557-582.

Levins, Richard. 2006. Strategies of abstraction. Biology \& Philosophy 21: 741755.

Levy, Arnon and William Bechtel. 2013. Abstraction and the organization of mechanisms. Philosophy of Science 80: 241-261.

Machamer, Peter, Lindley Darden, and Carl Craver. 2000. Thinking about mechanisms. Philosophy of Science 67: 1-25.

Miłkowski, Marcin. 2016. Explanatory completeness and idealization in large brain simulations: A mechanistic perspective. Synthese 193: 1457-1478.

Murray, J., E. Stanley, and D. Brown. 1986. On the spatial spread of rabies among foxes. Proceeding of the Royal Society of London. Series B: Biological Sciences 299: 111-150.

Musgrave, Alan. 1981. 'Unreal assumptions' in economic theory: The f-twist untwisted. Kyklos 34: 377-387.

Mäki, Uskali. 2000. Kinds of assumptions and their truth: Shaking an untwisted ftwist. Kyklos 53: 317-336.

Nathan, Marco. 2012. The varieties of molecular explanation. Philosophy of Science 79: 233-254.

Odenbaugh, Jay. 2005. Idealized, inaccurate but successful: A pragmatic approach to evaluating models in theoretical ecology. Biology \& Philosophy 20: 231255.

Paine, Robert. 1966. Food wed complexity and species diversity. American Naturalist 100: 65-75.

Persson, Lennart and Sebastian Diehl. 1990. Mechanistic individual-based approaches in the population/community ecology of fish. Annales Zoologi Fennica 27: 165-182.

Putnam, Hillary. 1975. Mind, language, and reality. Cambridge: Cambridge University Press.

Raerinne, Jani. 2011. Causal and mechanistic explanations in ecology. Acta Biotheoretica 59: 251-271.

Raerinne, Jani. 2013a. Explanatory, predictive, and heuristic roles of allometries and scaling relationships. BioScience 63: 191-198.

Raerinne, Jani. 2013b. Robustness and sensitivity of biological models. Philosophical Studies 166: 285-303.

Raerinne, Jani. Forthcoming. Explanations of exceptions in biology: Corrective asymmetry vs. autonomy. Synthese. doi: 10.1007/s11229-016-1195-9

Raerinne, Jani and Jan Baedke. 2015. Exclusions, explanations, and exceptions: On the causal and lawlike status of the competitive exclusion principle. Philosophy and Theory in Biology 7: 1-17. 
Rastetter, Edward, Anthony King, Bernard Cosby, George Hornberger, Robert O’Neill, and John Hobbie. 1992. Aggregating fine-scale ecological knowledge to model coarser-scale attributes to ecosystems. Ecological Applications 2(1): 55-70.

Reisman, Kenneth and Patrick Forber. 2005. Manipulation and the causes of evolution. Philosophy of Science 72: 1113-1123.

Schoener, Thomas. 1986. Mechanistic approaches to community ecology: A new reductionism? American Zoologist 26: 86-106.

Thulke, H.-H., V. Grimm, M. Müller, C. Staubach, L. Tischendorf, C. Wissel, and F. Jeltsch. 1999. From pattern to practice: A scaling-down strategy for spatially explicit modelling illustrated by the spread and control of rabies. Ecological Modelling 117: 179-202.

Tilman, David. 1977. Resource competition between planktonic algae: An experimental and theoretical approach. Ecology 58: 338-348.

Tilman, David. 1980. A graphical-mechanistic approach to competition and predation. American Naturalist 116: 362-393.

Tilman, David. 1986. A consumer-recourse approach to community ecology. American Zoologist 26: 5-22.

Tilman, David. 1990. Constraints and tradeoffs: Toward a predictive theory of competition and succession. Oikos 58: 3-15.

Vellend, Mark. 2010. Conceptual synthesis in community ecology. Quarterly Review of Biology 82: 183-206.

Weisberg, Michael and Kenneth Reisman. 2008. The robust Volterra principle. Philosophy of Science 75: 106-131.

Weslake, Brad. 2010. Explanatory depth. Philosophy of Science 77: 273-294.

Wimsatt, William. 1980. Randomness and perceived-randomness in evolutionary biology. Synthese 43: 287-339.

Wimsatt, William. 1986. Forms of aggregativity. In Human nature and natural knowledge, eds. Donogan, Alan, Anthony Perovich, and Michael Wedin, 259-291. Reidel: Dordrecht.

Winther, Rasmus. 2006. On the dangers of making scientific models ontologically independent: Taking Richard Levin's warning seriously. Biology \& Philosophy 21: 703-724.

Ylikoski, Petri and Jaakko Kuorikoski. 2010. Dissecting explanatory power. Philosophical Studies 148: 201-219. 